\title{
BERNOULLI PROPERTY OF SMOOTH EXTENSIONS OF BERNOULLI SHIFTS
}

Abstract. The paper gives a characterization of smooth extensions of Bernoulli shifts which have the Bernoulli property, i.e. their natural extensions to automorphisms are Bernoulli.

1. Introduction. Let $T_{i}, i=0,1$, be homeomorphisms of $I=[0,1]$ and let $\left(\Omega, \mathcal{B}, \mu_{p}, \sigma\right)$ be the one-sided Bernoulli shift where $\Omega=\{0,1\}^{\mathbb{N}}$ and $\mu_{p}$ is the $(p, q)$ measure. We denote by $\mathcal{A}$ the Borel $\sigma$-algebra of subsets of $I$ and by $\Lambda$ the Lebesgue measure on $I$. In the space $\Omega \times I$ we define the skew products

$$
\begin{aligned}
T(\omega, x) & =\left(\sigma(\omega), T_{\omega(0)}(x)\right), \\
S(\omega, x) & =\left(\sigma(\omega), S_{\omega(0)}(x)\right) \quad \text { where } \quad S_{i}=T_{i}^{-1}, i=0,1 .
\end{aligned}
$$

Let us denote by $M_{p}(S)$ the set of $S$-invariant probability measures $m$ on $\Omega \times I$ such that $m \mid \mathcal{B} \times\{\emptyset, I\}=\mu_{p}$. We define $M_{p}(T)$ in the same manner. The paper is motivated by the question of Benjamin Weiss about Bernoulli properties of extensions as above, posed during the conference: Dynamical Systems, Prague, 2015. The reason for this question is the open problem about existence of smooth $K$-automorphisms which are non-Bernoulli in dimension 3. We give a sufficient condition for the Bernoulli property to hold by using negativity of Lyapunov exponents (Theorem 2.3) and then by using the median method (Theorem 5.1). The main role in the proofs is

2010 Mathematics Subject Classification: Primary 37C40; Secondary 37A50.

Key words and phrases: smooth extension of one-sided Bernoulli shift, invariant product measure, Bernoulli property, Lyapunov exponent, supermartingale.

Received 11 July 2018; revised 2 February 2019.

Published online 8 April 2019. 
played by homeomorphisms defined below. Let

$$
\begin{aligned}
& \bar{T}(\varpi, x)=\left(\bar{\sigma}(\varpi), T_{\varpi(0)}(x)\right), \\
& \bar{S}(\varpi, x)=\left(\bar{\sigma}(\varpi), S_{\varpi(0)}(x)\right) .
\end{aligned}
$$

Here $\bar{\Omega}=\{0,1\}^{\mathbb{Z}}$ and $\bar{\sigma}$ is the two-sided $(p, q)$-Bernoulli shift. Then $\bar{T}$ and $\bar{S}^{-1}$ are topologically conjugate by $[8$, Theorem 1$]$. The conjugating transformation $\chi$ defines the map ${ }^{*}: M_{p}(T) \rightarrow M_{p}(S)$ by

$$
\mu^{*}(A)=\bar{\mu}\left(\chi\left(P^{-1}(A)\right)=P \circ \chi^{-1} \circ \bar{\mu}(A) \quad \text { for } A \in \mathcal{B} \times \mathcal{A} .\right.
$$

Here $P(\varpi, x)=(\omega, x)$ and $\bar{\mu}$ is the measure such that $(\bar{T}, \bar{\mu})$ is the natural extension of $(T, \mu)$ to an automorphism. The inverse map to * has the same properties. For a detailed description see [8].

DEFINITION 1.1. We say that a measure preserving transformation has the Bernoulli property if its natural extension to an automorphism is Bernoulli.

TheOREM 1.2. Let $T_{i}, i=0,1$, be homeomorphisms of $I$ and $\nu \in M_{p}(T)$. Then $(T, \nu)$ has the Bernoulli property if and only if $\left(S, \nu^{*}\right)$ does.

Proof. Let $(\bar{T}, \bar{\nu})$ be a Bernoulli automorphism. Hence $\left(\bar{S}^{-1}, \chi^{-1} \circ \bar{\nu}\right)$ is Bernoulli by topological conjugation of $\bar{T}$ with $\bar{S}^{-1}$ via $\chi$. Therefore $\left(\bar{S}, \chi^{-1} \circ \bar{\nu}\right)$ is Bernoulli too and it is the natural extension of $\left(S, \nu^{*}\right)$ to an automorphism by the properties of the inverse map to $*$.

Corollary 1.3. Let $\varphi: \Omega \rightarrow I$ be a measurable function which is T-invariant, i.e.

$$
T_{\omega(0)}(\varphi(\omega))=\varphi(\sigma(\omega)) \quad \text { for } \mu_{p} \text {-a.e. } \omega .
$$

Let $\nu \in M_{p}(T)$ be given by

$$
\nu(B \times A)=\mu_{p}\left(B \cap \varphi^{-1}(A)\right) \quad \text { for } B \in \mathcal{B}, A \in \mathcal{A} .
$$

Then $\left(S, \nu^{*}\right)$ has the Bernoulli property.

Proof. $(T, \nu)$ is measure-theoretically isomorphic to the one-sided $(p, q)$ Bernoulli shift. Here the isomorphic transformation $\psi$ is given by $\psi(\omega, \varphi(\omega))$ $=\omega$. Hence $(\bar{T}, \bar{\nu})$ is a Bernoulli automorphism and therefore $\left(S, \nu^{*}\right)$ has the Bernoulli property by Theorem 1.2.

We will apply the above to transformations from [9]. We also consider asymptotic properties of such skew products. Therefore the following result will be useful.

LEMmA 1.4. Let $T_{i}, i=0,1$, be homeomorphisms of $I$ such that $T_{0}(x)>x$ and $T_{1}(x)<x$ for $x \in(0,1)$. If $\mu_{p} \times \mu \in M_{p}(S)$ where $\mu(\{0\})=\mu(\{1\})=0$ then $\left(\mu_{p} \times \mu\right)^{*}$ is not a product measure. 
Proof. Assume on the contrary that $\left(\mu_{p} \times \mu\right)^{*}=\mu_{p} \times \nu$ for some measure $\nu$. Then

$$
\mu_{p} \times \nu\left(A_{i} \times B\right)=p_{i} \nu(B)=\mu_{p} \times \mu\left(A_{i} \times S_{i}(B)\right)=p_{i} \mu\left(S_{i}(B)\right), \quad i=0,1 .
$$

Here $A_{i}=\{\omega: \omega(0)=i\}$ and $B \in \mathcal{B}$. Hence $\mu(B)=\mu\left(T_{1}^{-1} \circ T_{0}(B)\right)$ for every $B \in \mathcal{B}$. Therefore $\mu=\delta_{\{0\}}$ or $\mu=\delta_{\{1\}}$, which yields a contradiction.

2. Lyapunov exponents method. Let $T_{i}, i=0,1$, be homeomorphisms of $I$ such that

$$
T_{i}=\left(1-\epsilon_{i}\right) x+\epsilon_{i} g(x), \quad i=0,1,
$$

for some reals $\epsilon_{0}<0, \epsilon_{1}>0$. Here $g$ is a $C^{1}[0,1]$ homeomorphism of $I$ with $g(x)<x$ for $x \in(0,1)$. The following result characterizes $M_{p}(S)$, Theorem 2.1].

TheOREM 2.1. If $\mu_{p} \times \mu \in M_{p}(S)$ is such that $\mu(\{0\})=\mu(\{1\})=0$ then the distribution function of $\mu$ is a homeomorphism of I. Moreover $\mu_{p} \times \mu$ is ergodic and

$$
M_{p}(S)=\operatorname{conv}\left\{\mu_{p} \times \delta_{\{0\}}, \mu_{p} \times \delta_{\{1\}}, \mu_{p} \times \mu\right\} .
$$

Let us denote

$$
\Lambda_{i}=p \log \left(T_{0}^{\prime}(i)\right)+(1-p) \log \left(T_{1}^{\prime}(i)\right) \quad \text { for } i=0,1,
$$

and let $\pi$ be the projection map $\pi(\omega, x)=x$. Here we assume that $T_{0}^{\prime}(i)>0$ and $T_{1}^{\prime}(i)>0$ for $i=0,1$. By using [2, Theorem 2.5] we get

Theorem 2.2. Assume $\Lambda_{i}<0$ for $i=0,1$. Then there exist measurable functions $\varphi_{i}: \Omega \rightarrow I, i=1,2$, which are $T$-invariant, $0<\varphi_{1}(\omega) \leq \varphi_{2}(\omega)$ $<1$ and for $\mu_{p}$-a.e. $\omega$,

- if $x<\varphi_{1}(\omega)$, then $\lim _{n \rightarrow \infty} \pi\left(T^{n}(\omega, x)\right)=0$,

- if $x>\varphi_{2}(\omega)$, then $\lim _{n \rightarrow \infty} \pi\left(T^{n}(\omega, x)\right)=1$.

Now we obtain the following result.

Theorem 2.3. Assume $\Lambda_{i}<0$ for $i=0,1$. Then:

(1) There exists a measurable function $\varphi: \Omega \rightarrow I$ which is T-invariant, $0<\varphi(\omega)<1$ and for $\mu_{p}$-a.e. $\omega$,

- if $x<\varphi(\omega)$, then $\lim _{n \rightarrow \infty} \pi\left(T^{n}(\omega, x)\right)=0$,

- if $x>\varphi(\omega)$, then $\lim _{n \rightarrow \infty} \pi\left(T^{n}(\omega, x)\right)=1$.

(2) $S$ has an invariant measure $\mu_{p} \times \nu$ such that the distribution function of $\nu$ is a homeomorphism of $I$ and the natural extension of $\left(S, \mu_{p} \times \nu\right)$ to an automorphism is Bernoulli.

Proof. Since $\Lambda_{i}<0$ for $i=0,1$, there exist measures

$$
\nu_{i}(B \times A)=\mu_{p}\left(B \cap \varphi_{i}^{-1}(A)\right) \quad \text { for } B \in \mathcal{B}, A \in \mathcal{A}, i=1,2 .
$$


Here $\nu_{i} \in M_{p}(T)$ and $\nu_{i}(\Omega \times\{0\})=\nu_{i}(\Omega \times\{1\})=0$ for $i=1,2$. The measures $\nu_{i}^{*} \in M_{p}(S), i=1,2$, have the same property. If we show that $\varphi_{1}(\omega)=\varphi_{2}(\omega)$ for $\mu_{p}$-a.e. $\omega$ then by Corollary 1.3 we get the assertion.

Therefore, we repeat the reasoning as above for $\bar{S}^{-1}$ instead of $T$. Then there exist two measures $\bar{\gamma}_{i}$ analogous to $\nu_{i}$ by Theorem 2.2 as $\bar{\Lambda}_{i}=\Lambda_{i}$ $<0$ for $i=0,1$. These measures are $\bar{S}^{-1}$-invariant and hence $\bar{S}$-invariant. By using [2, Theorem 4.2] we get $S$-invariant measures $\mu_{p} \times \eta_{i}$ such that $\eta_{i}(\{0\})=\eta_{i}(\{1\})=0$ and

$$
\mu_{p} \times \eta_{i}(C)=\bar{\gamma}_{i}\left(P^{-1}(C)\right) \quad \text { for } C \in \mathcal{B} \times \mathcal{A}, i=1,2 .
$$

Consequently, $\mu_{p} \times \eta_{1}=\mu_{p} \times \eta_{2}=\nu_{1}^{*}=\nu_{2}^{*}$ by Theorem 2.1. Therefore $\nu_{1}=\nu_{2}$ by the injectivity of $*$. Hence $\varphi_{1}(\omega)=\varphi_{2}(\omega)$ for $\mu_{p}$-a.e. $\omega$.

COROLlary 2.4. If $\Lambda_{0}<0$ and $\varphi_{1}(\omega)<1$ for $\mu_{p}$-a.e. $\omega$ then conclusion (2) of Theorem 2.3 holds.

In order to prove (1) we do not need the negativity of the Schwarzian derivative of $T_{i}, i=0,1$, which is assumed in [4], and we do not use the essential contraction property of $S$ assumed in [2. The above results have been presented during the conference: New Developments around $\times 2 \times 3$ Conjecture and other Classical Problems in Ergodic Theory, Cieplice, 2016.

3. Conclusions and examples. We give some examples which satisfy the assumptions of Theorem 2.3.

Corollary 3.1. If $\mu_{p} \times \Lambda$ is $S$-invariant and $g^{\prime}(0)<1, g^{\prime}(1)>1$ then $\left(S, \mu_{p} \times \Lambda\right)$ has the Bernoulli property.

Proof. $\mu_{p} \times \Lambda$ is $S$-invariant $\Leftrightarrow p \epsilon_{0}+(1-p) \epsilon_{1}=0$. Hence

$$
\begin{aligned}
\Lambda_{i} & =p \log \left(1-\epsilon_{0}+\epsilon_{0} g^{\prime}(i)\right)+(1-p) \log \left(1-\epsilon_{1}+\epsilon_{1} g^{\prime}(i)\right) \\
& <\log 1=0 \quad \text { for } i=0,1
\end{aligned}
$$

Let us consider a more general situation. We know that if $\mu_{p} \times \nu$ for $\nu \approx \Lambda$ is $S$-invariant then $\left(S, \mu_{p} \times \nu\right)$ has the K-property (see [8]). Below we show by an example that the Bernoulli property is possible for $\nu \neq \Lambda$.

EXAMPLE 3.2. Let

$$
T_{0}(x)=2 x-g(x) \quad \text { and } \quad T_{1}(x)=g(x)
$$

where $g$ is given by [9, Theorem 6.6] for $F(x)=\left(1-(1-x)^{2}\right)^{1 / 2}$ and some $p \in(1 / 2, \sqrt{2} / 2)$. Then $\mu_{p} \times \nu$, where $d \nu / d \Lambda=F^{\prime}$, is $S$-invariant. We show that $g \in C^{1}[0,1]$ and $\Lambda_{i}<0$ for $i=0,1$. Let us consider the continuity of $g^{\prime}$ at $0^{+}$. By [9, proof of Theorem 6.6] we have

$$
0<g(x)<\varphi(x)=2 x-F^{\prime-1}\left(\frac{1}{2 p} F^{\prime}(x)\right) \quad \text { for } x \in(0,1) \text {. }
$$


Since $\varphi_{+}^{\prime}(0)=2-(2 p)^{2}$ we have

$$
0 \leq \liminf _{x \rightarrow 0^{+}} \frac{g(x)}{x} \leq \limsup _{x \rightarrow 0^{+}} \frac{g(x)}{x} \leq 2-(2 p)^{2} .
$$

Simultaneously, by the identity

$$
F(x)=p F(2 x-g(x))+(1-p) F(g(x)) \quad \text { for } x \in[0,1]
$$

we get

$$
\lim _{x \rightarrow 0^{+}}\left[p\left(2-\frac{g(x)}{x}\right)^{1 / 2}+(1-p)\left(\frac{g(x)}{x}\right)^{1 / 2}\right]=1 .
$$

Therefore $\liminf \sin _{x \rightarrow 0^{+}} \frac{g(x)}{x}$ and $\lim \sup _{x \rightarrow 0^{+}} \frac{g(x)}{x}$ are solutions of the equation

$$
p(2-a)^{1 / 2}+(1-p) a^{1 / 2}=1 .
$$

The solutions are

$$
a_{1}=1 \quad \text { and } \quad a_{2}=\left(\frac{1-2 p^{2}}{1-2 p+2 p^{2}}\right)^{2}<1 .
$$

Hence $g_{+}^{\prime}(0)=a_{2}$ by (1). Moreover, by (2) we have

$$
\lim _{x \rightarrow 0^{+}} g^{\prime}(x)=\frac{\left(2-g_{+}^{\prime}(0)\right)^{1 / 2}-2 p}{(1-p)\left(\frac{2}{g_{+}^{\prime}(0)}-1\right)^{1 / 2}-p},
$$

which together with (3) implies $\lim _{x \rightarrow 0^{+}} g^{\prime}(x)=g_{+}^{\prime}(0)$.

We also obtain $\Lambda_{0}<0$ :

$$
\begin{aligned}
\frac{1}{2} \Lambda_{0} & =p \log \left(2-a_{2}\right)^{1 / 2}+(1-p) \log a_{2}^{1 / 2} \\
& <\log \left(p\left(2-a_{2}\right)^{1 / 2}+(1-p) a_{2}^{1 / 2}\right)=\log 1=0 .
\end{aligned}
$$

The proof of continuity of $g^{\prime}$ at $1^{-}$is similar. We use the inequality

$$
2 x-1<g(x)<\varphi(x) \quad \text { for } x \in(1 / 2,1)
$$

and the identity

$$
1-F(x)=p(1-F(2 x-g(x)))+(1-p)(1-F(g(x))) \quad \text { for } x \in[0,1] .
$$

Finally, we get $g_{-}^{\prime}(1)=4 p-1, \lim _{x \rightarrow 1^{-}} g^{\prime}(x)=g_{-}^{\prime}(1)$ and $\Lambda_{1}<0$.

REMARK 3.3. A similar result can be achieved by replacing $F$ by

$$
F_{\alpha}(x)=\left(1-(1-x)^{\alpha}\right)^{1 / \alpha} \quad \text { for } \alpha>1 .
$$

The same is also true for $F$ satisfying the assumptions of [9, Theorem 6.8].

4. The case of Lyapunov exponents $\Lambda_{0} \Lambda_{1} \leq 0$. It remains to analyse the case $\Lambda_{0} \Lambda_{1} \leq 0$. First assume that $\Lambda_{0} \Lambda_{1}<0$. 
THEOREM 4.1. If $\Lambda_{0}<0$ and $\Lambda_{1}>0$ then for every $x \in(0,1)$, $\lim _{n \rightarrow \infty} \pi\left(T^{n}(\omega, x)\right)=0 \quad$ and $\quad \lim _{n \rightarrow \infty} \pi\left(S^{n}(\omega, x)\right)=1 \quad$ for $\mu_{p}$-a.e. $\omega$.

Consequently,

$$
M_{p}(T)=M_{p}(S)=\operatorname{conv}\left\{\mu_{p} \times \delta_{\{0\}}, \mu_{p} \times \delta_{\{1\}}\right\} .
$$

Proof. By $T$-invariance of graph $\varphi_{1}$ and ergodicity of $\mu_{p}, \varphi_{1}(\omega)<1$ for a.e. $\omega$ or $\varphi_{1}(\omega)=1$ for a.e. $\omega$. We consider only the first case. By Corollary 2.4,

$$
M_{p}(S)=\operatorname{conv}\left\{\mu_{p} \times \delta_{\{0\}}, \mu_{p} \times \delta_{\{1\}}, \mu_{p} \times \mu\right\}
$$

for some measure $\mu$. By repeating the same reasoning for $S$ we get

$$
M_{p}(T)=\operatorname{conv}\left\{\mu_{p} \times \delta_{\{0\}}, \mu_{p} \times \delta_{\{1\}}, \mu_{p} \times \nu\right\}
$$

where $\mu_{p} \times \nu=\left(\mu_{p} \times \mu\right)^{*}$, which contradicts Lemma 1.4.

For $\Lambda_{0} \Lambda_{1}=0$ we apply martingale methods as in [5, proof of Theorem 5.1]. Let $x \in(0,1)$. Define two processes:

$x_{0}=x, \quad x_{n+1}=T_{\omega(n)}\left(x_{n}\right) \quad$ and $\quad u_{n}=-\ln \left(x_{n}\right) \quad$ for $n=0,1, \ldots$

Theorem 4.2. Assume $\Lambda_{0}=0$. If $T_{0}^{p}(x) T_{1}^{1-p}(x) \geq x$ for every $x \in I$ then the process $u_{n}$ is a supermartingale and hence

$$
\lim _{n \rightarrow \infty} x_{n}=1 \quad \text { for } \mu_{p} \text {-a.e. } \omega .
$$

Proof. Write $T_{i}(x)=c_{i} x\left(1+t_{i}(x)\right)^{-1}$ where $T_{i}^{\prime}(0)=c_{i}$ for $i=0,1$. Then

$$
u_{n+1}=-\ln \left(c_{\omega(n)}\right)+u_{n}+\ln \left(1+t_{\omega(n)}\left(e^{-u_{n}}\right)\right) .
$$

As $u_{n}$ is a Markov process it suffices to show that $E\left(u_{n+1} \mid u_{n}=u\right) \leq u$ for every $u \geq 0$. Indeed,

$$
\begin{aligned}
E\left(u_{n+1} \mid u_{n}=u\right)-u & =\int_{\Omega}-\ln \left(c_{\omega(n)}\right)+\ln \left(1+t_{\omega(n)}\left(e^{-u}\right)\right) d \mu_{p} \\
& =\int_{\Omega} \ln \left(1+t_{\omega(n)}\left(e^{-u}\right)\right) d \mu_{p} \\
& =p \ln \left(1+t_{0}\left(e^{-u}\right)\right)+(1-p) \ln \left(1+t_{1}\left(e^{-u}\right) \leq 0\right.
\end{aligned}
$$

because $T_{0}^{p}(x) T_{1}^{1-p}(x) \geq x$ for every $x \in I$. Here we use the equality

$$
\int_{\Omega} \ln \left(c_{\omega(n)}\right) d \mu_{p}=0 \quad \text { since } \Lambda_{0}=0 .
$$

Hence $u_{n}$ is a nonnegative supermartingale and by Doob's supermartingale convergence theorem (see [10, Section VII.4])

$$
\lim _{n \rightarrow \infty} u_{n}<\infty \text { for } \mu_{p^{-}} \text {a.e. } \omega \text {. }
$$


If $u_{n} \rightarrow u_{\infty}$ then $x_{n} \rightarrow x_{\infty} \in(0,1]$. From $T_{i}\left(x_{\infty}\right)=x_{\infty}$ for $i=0,1$ we get $x_{\infty}=1$.

In the case $\Lambda_{1}=0$ we define the process

$$
v_{n}=-\ln \left(1-x_{n}\right) \quad \text { for } n=0,1, \ldots
$$

Theorem 4.3. Assume $\Lambda_{1}=0$. If $\left(1-T_{0}(x)\right)^{p}\left(1-T_{1}(x)\right)^{1-p} \geq 1-x$ for every $x \in I$ then the process $v_{n}$ is a supermartingale and hence

$$
\lim _{n \rightarrow \infty} x_{n}=0 \quad \text { for } \mu_{p} \text {-a.e. } \omega .
$$

Proof. Write $T_{i}(x)=1-a_{i}(1-x)\left(1+r_{i}(1-x)\right)^{-1}$ where $T_{i}^{\prime}(1)=a_{i}$ for $i=0,1$ and repeat the reasoning in the proof of Theorem 4.2.

Corollary 4.4. If $\Lambda_{0}=0, p=1 / 2$ and $g^{\prime}(0)=\inf \left\{g^{\prime}(x): x \in I\right\}$ then (1) for every $x \in(0,1], \lim _{n \rightarrow \infty} \pi\left(T^{n}(\omega, x)\right)=1$ for $\mu_{0.5}$-a.e. $\omega$.

(2) $M_{0.5}(T)=M_{0.5}(S)=\operatorname{conv}\left\{\mu_{p} \times \delta_{\{0\}}, \mu_{p} \times \delta_{\{1\}}\right\}$.

Proof. Let $T_{0}=\left(1+\epsilon_{0}\right) x-\epsilon_{0} g(x)$ and $T_{1}=\left(1-\epsilon_{1}\right) x+\epsilon_{1} g(x)$ for some $\epsilon_{0}, \epsilon_{1}>0$. Since $\Lambda_{0}=0, p=1 / 2$ we have

$$
1+\epsilon_{0}\left(1-g^{\prime}(0)\right)=\frac{1}{1-\epsilon_{1}\left(1-g^{\prime}(0)\right)} .
$$

Hence

$$
\begin{aligned}
T_{0}(x) T_{1}(x) & =\left(x+\epsilon_{0}(x-g(x))\left(x-\epsilon_{1}(x-g(x))\right.\right. \\
& =x^{2}+\left(\epsilon_{0}-\epsilon_{1}\right) x(x-g(x))-\epsilon_{0} \epsilon_{1}(x-g(x))^{2} \geq x^{2}
\end{aligned}
$$

if and only if

$$
g^{\prime}(0) \leq \frac{g(x)}{x}
$$

for every $x \in(0,1)$. The assumptions of the theorem and the results of Theorem 4.2 give the conclusion.

By using the same reasoning we get

Corollary 4.5. If $\Lambda_{1}=0, p=1 / 2$ and $g^{\prime}(1)=\sup \left\{g^{\prime}(x): x \in I\right\}$ then

(1) for every $x \in[0,1) \lim _{n \rightarrow \infty} \pi\left(T^{n}(\omega, x)\right)=0$ for $\mu_{0.5}$-a.e. $\omega$,

(2) $M_{0.5}(T)=M_{0.5}(S)=\operatorname{conv}\left\{\mu_{p} \times \delta_{\{0\}}, \mu_{p} \times \delta_{\{1\}}\right\}$.

For a continuous family of fiber maps where $\Lambda_{0}=0$ or $\Lambda_{1}=0$, the sequence $\pi\left(T^{n}(\omega, x)\right)$ can be more complicated (see [7]).

5. Median method. By using the median method of Hans Crauel as in [3, proof of Theorem 1.8.4(iv)] we get 
THEOREM 5.1. Let $T_{i}$ be homeomorphisms of $I$ such that $T_{i}(0)=0$ and $T_{i}(1)=1$ for $i=0,1$. If $\bar{\nu} \in M_{p}(\bar{T})$ is an ergodic measure then there exists a measurable function $\varphi: \bar{\Omega} \rightarrow I$ which is $\bar{T}$-invariant and

$$
\bar{\nu}(B \times A)=\overline{\mu_{p}}\left(B \cap \varphi^{-1}(A)\right) \quad \text { for } B \in \overline{\mathcal{B}}, A \in \mathcal{A} .
$$

Therefore $(\bar{T}, \bar{\nu})$ is a Bernoulli automorphism.

The same conclusion can be obtained under weaker assumptions. In [1], the $T_{i}$ are logistic maps (therefore not invertible). In [6], $I$ is a compact manifold.

COROllary 5.2. If $T_{i}$ are homeomorphisms of $I$ such that $T_{i}(0)=0$ and $T_{i}(1)=1$ for $i=0,1$ and $\nu \in M_{p}(T)$ is an ergodic measure then $(T, \nu)$ has the Bernoulli property.

Corollary 5.2 can be directly applied to skew products considered in [2], [4], 5] and in our paper. Theorem 5.1 gives a partial positive answer to the question of Benjamin Weiss.

\section{References}

[1] N. Abbasi, M. Gharaei and A. J. Homburg, Iterated function systems of logistic maps: synchronization and intermittency, Nonlinearity 31 (2018), 3880-3913.

[2] L. Alsedà and M. Misiurewicz, Random interval homeomorphisms, Publ. Mat. 58 (2014), 15-36.

[3] L. Arnold, Random Dynamical Systems, Springer, Berlin, 1998.

[4] A. Bonifant and J. Milnor, Schwarzian derivatives and cylinder maps, in: Holomorphic Dynamics and Renormalization, Fields Inst. Comm. 53, Amer. Math. Soc., Providence, RI, 2008, 1-21.

[5] M. Gharaei and A. J. Homburg, Random interval diffeomorphisms, Discrete Contin. Dynam. Systems Ser. S 10 (2017), 241-272.

[6] A. J. Homburg, Synchronization in minimal iterated function systems on compact manifolds, Bull. Braz. Math. Soc. (N.S.) 49 (2018), 615-635.

[7] A. J. Homburg and V. F. Rabodonandrianandraina, On-off intermittency and chaotic walks, Ergodic Theory Dynam. Systems (online, 2019).

[8] Z. S. Kowalski, Dual skew products, genericity of the exactness property and finance, Int. J. Bifurcation Chaos Appl. Sci. Engrg. 21 (2011), 545-550.

[9] Z. S. Kowalski, Stability of smooth extensions of Bernoulli shifts, Appl. Math. (Warsaw) 44 (2017), 85-104.

[10] A. N. Shiryayev, Probability, Springer, 1984.

Zbigniew S. Kowalski

Faculty of Pure and Applied Mathematics

Wrocław University of Science and Technology

Wybrzeże Wyspiańskiego 27

50-370 Wrocław, Poland

E-mail: Zbigniew.Kowalski@pwr.edu.pl 